\title{
Elementos genéticos móviles asociados con resistencia a eritromicina en aislamientos de Streptococcus pneumoniae en Colombia
}

\author{
Viviana Ramos ${ }^{1}$, Carolina Duarte ${ }^{2}$, Aleyda Díaz ${ }^{3}$, Jaime Moreno ${ }^{1}$ \\ 1 Grupo de Microbiología, Subdirección de Investigación Científica y Tecnológica, Instituto Nacional de Salud, \\ Bogotá, D. C., Colombia \\ 2 Grupo de Microbiología, Subdirección de Red Nacional de Laboratorios, Instituto Nacional de Salud, Bogotá, D. C., \\ Colombia \\ ${ }^{3}$ Facultad de Ciencias Básicas, Universidad del Tolima, Ibagué, Tolima, Colombia
}

Introducción. La resistencia a macrólidos está aumentando en Streptococcus pneumoniae y se ha asociado con la presencia de elementos genéticos móviles.

Objetivo. Determinar la distribución de transposones en aislamientos invasivos de S. pneumoniae resistentes a eritromicina en Colombia entre 1994 y 2011.

Materiales y métodos. Se analizaron 225 aislamientos de $S$. pneumoniae resistentes a eritromicina. Los aislamientos tenían datos epidemiológicos, serotipo y sensibilidad antimicrobiana. Se determinaron los fenotipos con la prueba de doble disco y la presencia de transposones por medio de dos PCR múltiples para identificar los genes erm(B), mef(A), mef(E), tetM, cat, Aph3-III, int-Tn916, xis-Tn916, TnpA-Tn917, TnpR-Tn917 e int-Tn5252. Las relaciones 'clonales' se establecieron por electroforesis en campo pulsado.

Resultados. Del total de aislamientos, 62,7, 2,7 y 34,6\% presentaron fenotipos $\mathrm{cMLS}_{\mathrm{B}}$, iMLS $\mathrm{B}_{\mathrm{B}}$ y M, respectivamente. Se observó multirresistencia en $69,3 \%$ de estos aislamientos. Los elementos genéticos más comunes en aislamientos con fenotipo $\mathrm{MLS}_{\mathrm{B}}$ fueron: Tn5253 (34\%), principalmente en aislamientos con serotipo 6B relacionado con el clon España ${ }^{6 B}$-ST90; Tn 1545(18,4\% ), en aislamientos con el serotipo 19A relacionado con ST276, y Tn3872 (14,9\%), en aislamientos con serotipo 6B y 14. Otros siete transposones $(32,7 \%)$ se asociaron con diferentes serotipos. El elemento mega (Macrolide Efflux Genetic Assembly) (62,8 \%) se asoció con el serotipo 6A y el ST473 en aislamientos con fenotipo $\mathrm{M}$.

Conclusiones. La mayoría de los aislamientos de $S$. pneumoniae resistentes a eritromicina en Colombia presentaron el fenotipo $\mathrm{CMLS}_{\mathrm{B}}$ y se asociaron con la presencia de transposones que contienen determinantes de resistencia a otros antibióticos. Además, los aislamientos con fenotipo M contienen el gen mef(E) en el elemento mega.

Palabras clave: Streptococcus pneumoniae, eritromicina, resistencia a medicamentos, retroelementos, Colombia.

doi: http://dx.doi.org/10.7705/biomedica.v34i0.1684

\section{Mobile genetic elements associated with erythromycin-resistant isolates of Streptococcus pneumoniae in Colombia}

Introduction: Resistance to macrolide antibiotics is increasing in clinical isolates of Streptococcus pneumoniae and is associated with the presence of mobile genetic elements.

Objetive: The aim of this study was to determine the distribution of serotypes and transposons among macrolide-resistant invasive isolates of $S$. pneumoniae in Colombia from 1994 to 2011.

Materials and methods: A total of 225 macrolide-resistant $S$. pneumoniae isolates were analyzed. The isolates had epidemiological data, serotyping and antimicrobial susceptibility patterns. The phenotypes were tested by erythromycin-clindamycin double-disk test. We investigated the presence of transposons by several multiplex PCRs to identify the genes erm(B), mef(A), mef(E), tetM, Cat, Aph3-III, int-Tn916, xis-Tn916, TnpA-Tn917, TnpR-Tn917 and int-Tn5252.

Results: Of all isolates, $62.7 \%, 2.7 \%$ and $34.6 \%$ of isolates exhibited $\mathrm{CMLS}_{\mathrm{B}}$, iMLS $_{\mathrm{B}}$, and $\mathrm{M}$ phenotypes, respectively. Multiresistance was observed in $69.3 \%$ of these strains. The most prevalent genetic elements in $\mathrm{MLS}_{\mathrm{B}}$ were the Tn5253(34\%), mostly carried in serotype 6B isolates and found to be related

Contribución de los autores:

Jaime Moreno: diseño del estudio.

Carolina Duarte: pruebas fenotípicas.

Viviana Ramos y Aleyda Díaz: pruebas genotípicas.

Todos los autores participaron en el análisis de datos y la escritura del manuscrito. 
to the international clone Spain-ST90; Tn 1545 (18.4\%), carried by serotype 19A and related to ST276, and Tn3872 (14.9\%), mainly detected in capsular types 6B and 14 . Other seven transposons $(32.7 \%)$ were associated with different serotypes. The mega element (62.8\%) was associated to serotype $6 \mathrm{~A}$ and ST473 in M phenotype.

Conclusions: The majority of erythromycin-resistance isolates of $S$. pneumoniae in Colombia had the $\mathrm{CMLS}_{\mathrm{B}}$ phenotype and was associated with the presence of transposons, which carry multiple resistance determinants for other antibiotics. Moreover, isolates with $M$ phenotype carried the gene $\operatorname{mef}(\mathrm{E})$ in the mega element.

Key words: Streptococcus pneumoniae, erythromycin, drug resistance, retroelements, Colombia. doi: http://dx.doi.org/10.7705/biomedica.v34i0.1684

Streptococcus pneumoniae es una causa importante de morbilidad y mortalidad para el hombre, así como de infecciones como la sinusitis, la otitis media, la neumonía y la meningitis (1). El tratamiento con antibióticos puede verse afectado por la resistencia a estos fármacos, especialmente a betalactámicos y macrólidos, limitando así las opciones de tratamiento antimicrobiano efectivo (2).

La resistencia a macrólidos en $S$. pneumoniae se ha incrementado durante los últimos años a nivel mundial y está mediada principalmente por dos mecanismos: la modificación del sitio blanco por metilación del $A R N r 23 S$ codificada por el gen $\operatorname{erm}(\mathrm{B})$ y relacionado con el fenotipo $\mathrm{MLS}_{\mathrm{B}}$ (resistencia a macrólidos, lincosamidas y estreptograminas de tipo B) y el mecanismo de bomba de expulsión de macrólidos codificado por los genes mef y relacionado con el fenotipo $M$ (resistencia a macrólidos de 14 y 15 carbonos). En algunos casos, la resistencia a macrólidos también puede explicarse por las mutaciones en el gen $A R N r 23 S$ o las alteraciones en las proteínas ribosómicas L4 y L22 (3).

La dispersión de la resistencia a la eritromicina se ha asociado con la presencia de elementos genéticos móviles, como los transposones, que facilitan la transferencia de los mecanismos de resistencia (4). En S. pneumoniae la resistencia por metilación de la subunidad ribosómica 235 está asociada con la inserción del gen erm(B) en un transposón de conjugación de la familia Tn916, el cual contiene el gen de resistencia a tetraciclina (tetM) y los genes de la integrasa (int) y excisasa (xis) que lo capacitan para realizar su propia transposición. Los transposones de esta familia incluyen el Tn6002, el

\section{Correspondencia:}

Jaime Moreno, Grupo de Microbiología, Subdirección de Investigación Científica y Tecnológica, Instituto Nacional de Salud, Avenida calle 26 № 52-20, Bogotá, D. C., Colombia. Teléfono: (571) 2207700 , extensión 1421

jmoreno@ins.gov.co

Recibido: 05/06/13; aceptado: 07/01/14
Tn1545 (que transporta el gen de resistencia a kanamicina, aphA3), el Tn3872, el cual resulta de la inserción del Tn917 que, a su vez, contiene los genes TnpA (transposasa) y $\operatorname{TnpR}$ (resolvasa) en el transposón Tn916, y el Tn5253, un transposón compuesto conformado por la inserción del Tn5251 de la familia Tn916 en el elemento genético Tn5252, el cual transporta el marcador de resistencia a cloranfenicol (cat) y la integrasa int5252 (4).

El mecanismo de la bomba de expulsión de macrólidos en $S$. pneumoniae es codificado por tres subclases de gen mef: $\operatorname{mef}(\mathrm{E}), \operatorname{mef}(\mathrm{A})$ y $\operatorname{mef}(\mathrm{I})$. El gen $\operatorname{mef}(\mathrm{E})$ es transportado por el ensamblaje genético del expulsión de macrólidos, o elemento mega, y se ha descrito en dos transposones compuestos de la familia Tn916: el Tn2009, que transporta el gen tetM, y el Tn2010, que contiene los genes tetM, erm $(\mathrm{B})$ y mef(E). En $S$. pneumoniae el único elemento genético móvil reconocido que transporta el gen $\operatorname{mef}(\mathrm{A})$ es el transposón defectuoso Tn1207.1 (4).

En Colombia, la vigilancia nacional por el laboratorio como parte del proyecto del Sistema de Redes de Vigilancia de los Agentes Bacterianos Causantes de Neumonías y Meningitis (SIREVAll) ha mostrado el incremento progresivo de la resistencia a eritromicina, la cual pasó de 2,6 \% en 1994 a 14,5 \% en el 2012 (5); sin embargo, se desconocen los elementos genéticos móviles que están involucrados en la diseminación de esta resistencia.

El objetivo de este estudio fue determinar la distribución de los principales elementos genéticos móviles en aislamientos invasivos de $S$. pneumoniae resistentes a eritromicina en Colombia entre 1994 y 2011.

\section{Materiales y métodos}

\section{Aislamientos}

Se analizaron 225 aislamientos de S. pneumoniae resistentes a eritromicina recuperados de pacientes 
con enfermedad invasiva entre 1994 y 2011 mediante el programa de vigilancia del proyecto SIREVA II de la Organización Panamericana de la Salud (OPS), y remitidos al Grupo de Microbiología del Instituto Nacional de Salud por los laboratorios de salud pública del país.

Se definieron como aislamientos invasivos a los aislamientos de $S$. pneumoniae recuperados de sitios corporales normalmente estériles, tales como sangre, líquido cefalorraquídeo u otro fluido corporal (líquido pleural, líquido peritoneal, etc.). Los aislamientos provenían principalmente de Bogotá (34,2\%), Antioquia (34,2\%), Valle (14,2\%), Risaralda (3,6\%), Santander (3,1\%), y los restantes $(10,7 \%)$ de otros nueve departamentos.

La información de serotipo, sensibilidad a antimicrobianos, genes de resistencia a eritromicina y 'clonalidad' de los aislamientos recolectados de 1994 a 2008 ya había sido publicada $(6,7)$. En el presente estudio se identificó en estos mismos aislamientos la clase de gen mef y los elementos genéticos móviles asociados con la resistencia a macrólidos.

\section{Sensibilidad antimicrobiana}

Los aislamientos de $S$. pneumoniae se identificaron por morfología de la colonia, pruebas de solubilidad en la bilis y sensibilidad a la optoquina. Los aislamientos contaban con los datos de serotipo por reacción de Quellung (Statens Serum Institut, Copenhague, Dinamarca) y sensibilidad antimicrobiana a penicilina, eritromicina, cloranfenicol, trimetoprim-sulfametoxazol, tetraciclina, ceftriaxona y vancomicina, de acuerdo con las técnicas estandarizadas a nivel regional $(8,9)$.

La multirresistencia se definió como resistencia a tres o más familias de antibióticos. Para determinar el fenotipo de resistencia a los macrólidos constitutivo $\left(\mathrm{CMLS}_{\mathrm{B}}\right.$ ), inducible ( $\mathrm{iMLS}_{\mathrm{B}}$ ) o fenotipo $\mathrm{M}$ de los aislamientos del periodo 2009 a 2011, se empleó la prueba de doble disco (10) y se determinó la concentración inhibitoria mínima (CIM) a clindamicina por el método de microdilución en caldo (11).

\section{Detección de genes de resistencia y genes relacionados con transposones}

Los genes de resistencia a macrólidos erm(B) y mef se detectaron por medio de la técnica de reacción en cadena de la polimerasa (PCR) $(7,12)$. La diferenciación de las subclases mef(A) y mef $(\mathrm{E})$ e intTn5252 se realizó por PCR utilizando los iniciadores y condiciones descritas por Shiojima, et al. (13).
Los genes relacionados con los transposones Tn916 y Tn917 (int-Tn916, xis-Tn916, TnpA-Tn917, y TnpR-Tn917) y los genes de resistencia a tetraciclina (tet M), cloranfenicol (cat) y kanamicina (aph3-III) se detectaron por la prueba PCR múltiple utilizando iniciadores previamente descritos (14-17) y estandarizada en el laboratorio con las siguientes concentraciones de reactivos: solución tampón 1X, dNTP $400 \mathrm{nM}$, dos unidades de polimerasa Taq, $3 \mathrm{mM}$ de $\mathrm{MgCl}_{2}$ con dos mezclas de iniciadores: mezcla uno con los genes (int 100nM, xis 200nM y cat 100nM) y mezcla dos (TnpA $308 \mathrm{nM}, \operatorname{TnpR}$ $154 \mathrm{nM}$, tetM $30 \mathrm{nM}$ y aph3-III 308nM) en un volumen final de reacción de $25 \mu$ l. Las condiciones del termociclador constaron de un ciclo inicial de $95{ }^{\circ} \mathrm{C}$ por un minuto, seguido por 35 ciclos de $95^{\circ} \mathrm{C}$ por 30 segundos, de $53{ }^{\circ} \mathrm{C}$ por un minuto y 30 segundos y de $72{ }^{\circ} \mathrm{C}$ por dos minutos, con un ciclo final de $72{ }^{\circ} \mathrm{C}$ por un minuto.

Para identificar los diferentes transposones se utilizaron las siguientes combinaciones de genes relacionados con transposones y resistencia a antibióticos: Tn6002 [erm(B), tetM, int-Tn916 y xis-Tn916], Tn3872 [erm(B), tetM, TnpA-Tn917 y TnpR-Tn917], Tn 1545 [erm(B), tetM, int-Tn916, xisTn916, y aph3 III], Tn5253 [erm(B), tetM, int-Tn916, xis-Tn916, cat e int5252], elemento mega [mef(E)], Tn2009 [mef(E), tetM, int-Tn916 y xis-Tn916], Tn2010 [erm(B), mef(E), tetM, int-Tn916 y xisTn916], Tn1207.1 [mef(A)] e Tn1116 [erm(B), tetM] $(4,17)$ (cuadro suplementario 1. Disponible en: doi: http://dx.doi.org/10.7705/biomedica.v34i0.1684)

\section{Tipificación molecular}

La tipificación molecular de los aislamientos resistentes a eritromicina recolectados del 2009 al 2011 se realizó mediante electroforesis en gel de campo pulsado (PFGE) después de la restricción con la enzima Smal (18). Los patrones de PFGE de 195 aislamientos se compararon con los de los clones España ${ }^{23 F_{-}}$T81, España ${ }^{6 B}-S T 90$, España ${ }^{9 V_{-}}$-ST156 y Colombia ${ }^{23 F}$-ST338 y las secuencias tipo ST276, ST320 y ST473, identificados durante la vigilancia molecular de aislamientos de $S$. penumoniae con sensibilidad disminuida a penicilina realizada por el grupo. Además, se incluyó la información sobre la 'clonalidad' de 30 aislamientos previamente reportada (7).

\section{Resultados}

El análisis de la información de los 225 aislamientos resistentes a eritromicina permitió determinar que $37,8 \%$ de los aislamientos provenía de niños 
menores de 2 años, 12,9\% del grupo de 2 a 6 años, $4 \%$ del de 6 a 14 años, $20 \%$ del grupo de mayores de 14 a 50 años y $25,3 \%$ del de mayores de 50 años.

Del total de aislamientos, 40,9\% se recuperó de pacientes con diagnóstico de neumonía; $20 \%$, de meningitis; $21,3 \%$, de sepsis, y $17,8 \%$, de otros diagnósticos; $18,7 \%$ de los aislamientos recuperados de pacientes que no tenían diagnóstico de meningitis y 3,1\% de aquellos pacientes con meningitis fue resistente a penicilina; 56,4 \% tenía resistencia a tetraciclina, $46,7 \%$ a trimetoprim-sulfametoxazol y $30,6 \%$ a cloranfenicol; todos los aislamientos fueron sensibles a la vancomicina. La multirresistencia se observó en $69,3 \%$ de los aislamientos. Los principales serotipos identificados fueron $6 \mathrm{~B}$ (27,1\%), 19A (18,2\%), 6A (16,4\%), 14 (12,4\%), $19 \mathrm{~F}(6,6 \%)$ y $23 \mathrm{~F}(5,3 \%)$.

El fenotipo $M_{L} S_{B}$ se expresó en $65,3 \%(n=147)$ de los aislamientos, de los cuales $95,9 \%$ fue constitutivo y $4,1 \%$ mostró fenotipo de resistencia inducible; el gen erm(B) se detectó en $98 \%$ de estos aislamientos y 16 de ellos tenían los genes $\operatorname{erm}(\mathrm{B})$ y $\operatorname{mef}(\mathrm{E})$.

En $93,1 \%$ de los aislamientos con fenotipo $\mathrm{MLS}_{\mathrm{B}}$ se identificaron transposones. El elemento genético móvil detectado con mayor frecuencia fue el Tn5253 (34\%), transportado principalmente en aislamientos con serotipo 6B (70\%) y relacionado genéticamente con el clon internacional España ${ }^{6 B_{-}}$ ST90. El Tn1545 se identificó en 18,4\% de los aislamientos, principalmente con el tipo capsular $19 \mathrm{~A}(51,8 \%)$ y se relacionó con el ST276, que es una variante del complejo 'clonal' CC230. El 14,9\% de los aislamientos transportaba el Tn3872, distribuido principalmente en aislamientos con serotipos $6 \mathrm{~B}(40,1 \%), 14(22,7 \%)$ y $23 \mathrm{~F}(22,7 \%)$ y relacionados con los clones España ${ }^{23 F}-S T 81$, España $^{6 B_{-}}$ST90, España ${ }^{9 \mathrm{~V}}$-ST156 y Colombia ${ }^{23 F_{-}}$ ST338. El Tn6002 se determinó en $11,6 \%$ de los aislamientos, especialmente con el tipo capsular 14 (52,9\%), y el Tn2010 se identificó en los aislamientos que presentaban resistencia dual a eritromicina $(10,8 \%)$, incluidos 15 aislamientos con el tipo capsular 19A relacionados con el ST320, y un aislamiento 19F relacionado con el España ${ }^{9 \mathrm{~V}}-\mathrm{ST} 156$. Otros transposones identificados en aislamientos con fenotipo $\mathrm{MLS}_{B}$ fueron el Tn1116 (4,1\%)y el SpnAp3ermB $(2,0 \%)$, en tanto que en $4,2 \%$ de los aislamientos no se identificaron transposones con la metodología empleada en este estudio (cuadro 1, figura suplementaria 1. Disponible en: doi: http://dx.doi. org/10.7705/biomedica.v34i0.1684)

El fenotipo $M$ se observó en $34,7 \%(n=78)$ de los aislamientos, de los cuales 69 (30,6\%) tenían el gen $\operatorname{mef}(\mathrm{E})$, dos tenían el gen $\operatorname{mef}(\mathrm{A})$ y siete no amplificaron para el gen mef. En $62,8 \%$ de estos aislamientos estaba presente el elemento mega, principalmente en aislamientos con serotipo 6A $(55,1 \%)$ relacionados con el ST473. El Tn2009 se identificó en $17,9 \%$ de los aislamientos con serotipos 19F, 6B y 19A. En un aislamiento con el determinante de resistencia mef(A) se determinó el Tn1207.1. En el resto de los aislamientos (18,1\%) no se pudieron identificar elementos genéticos móviles relacionados con resistencia a eritromicina (cuadro 1, figura suplementaria 1).

\section{Discusión}

Los resultados de este estudio demuestran que la resistencia a macrólidos en aislamientos invasivos colombianos de $S$. pneumoniae ha sido consecuencia principalmente de la dispersión de clones internacionales que han adquirido transposones con marcadores de resistencia a varios antibióticos. Además, se estableció que el subtipo $\operatorname{mef}(\mathrm{E})$ fue el determinante de resistencia en aislamientos que exhibieron el fenotipo de resistencia $\mathrm{M}$ y que su dispersión se debió a la transferencia del elemento mega.

La neumonía fue el principal diagnóstico asociado con aislamientos resistentes a eritromicina. En América Latina la incidencia de neumonía adquirida en la comunidad es de 919/100.000 niños menores de cinco años, con S. pneumoniae como el principal agente etiológico (19), mientras que en Colombia, Benavidez, et al., encontraron para Bogotá una incidencia de neumonía bacteriana de 54,2/100.000 niños menores de 36 meses (20). Para tratar estas infecciones, varias asociaciones médicas colombianas han recomendado el tratamiento combinado con un betalactámico y un macrólido (21). Teniendo en cuenta que 69,3\% de los aislamientos resistentes a eritromicina presentó mutirresistencia, la dispersión de aislamientos resistentes a eritromicina puede limitar la efectividad del tratamiento empírico y las opciones terapéuticas.

$\mathrm{Al}$ igual que en estudios previos, erm(B) es el determinante genético más frecuente en los aislamientos colombianos de $S$. pneumoniae resistentes a la eritromicina $(6,7)$. El mecanismo de expulsión 


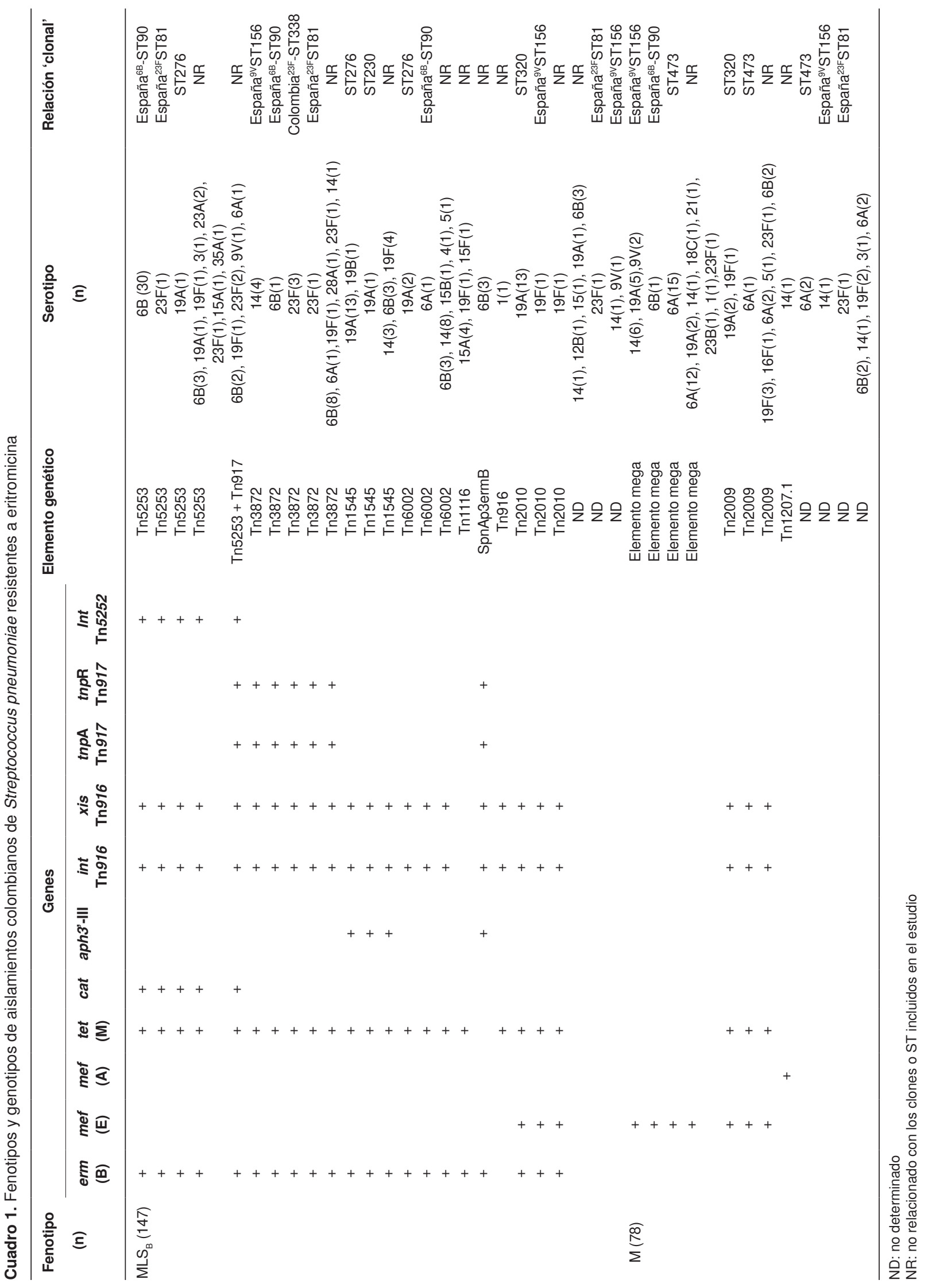


de macrólidos es conferido por proteínas codificadas por los genes $\operatorname{mef}(\mathrm{A}), \operatorname{mef}(\mathrm{E})$ y $\operatorname{mef}(\mathrm{I})(4,22)$, los cuales otorgan diferencias genéticas distintivas a los aislamientos; por lo tanto, al describir la epidemiología de la resistencia a los macrólidos es importante diferenciar estos genes, ya que esto podría contribuir a una mejor comprensión de la propagación de la resistencia a eritromicina en S. pneumoniae (23). Como resultado de esta diferenciación se pudo establecer que $\operatorname{mef}(\mathrm{E})$ es el principal determinante de la resistencia en los aislamientos estudiados con fenotipo $\mathrm{M}$.

El principal elemento genético móvil identificado en los asilamientos colombianos de $S$. pneumoniae resistentes a la eritromicina fue el transposón de conjugación Tn5253, transposón compuesto que consiste en Tn916, el cual se inserta en el Tn5252 y está asociado con resistencia a tetraciclina y cloranfenicol (17). HendersonBegg, et al., realizaron el análisis de los genes de este transposón y los datos de antibiograma, encontrando un alto grado de variación de los elementos genéticos relacionados con Tn5253 en aislamientos clínicos de $S$. pneumoniae. Además, establecieron que este transposón es común en clones internacionales de $S$. pneumoniae resistentes a antimicrobianos, incluido el clon España ${ }^{6 B_{-}}$ ST90 (24). En el presente estudio, la asociación de este clon con Tn5253 se estableció en 49,2\% de los aislamientos con tipo capsular 6B.

El Tn1545 se identificó en $18,4 \%$ de los aislamientos estudiados; este transposón fue el primer elemento genético identificado en aislamientos clínicos de $S$. pneumoniae multirresistentes que portaban el gen $\operatorname{erm}(\mathrm{B})$ y los determinantes de resistencia a tetraciclina, kanamicina y aminoglucósidos (25). La presencia de este transposón explica las tasas de resistencia a tetraciclina y eritromicina observadas en Italia (26) y España (27), y los aislamientos con este transposón se han relacionado con los clones Dinamarca ${ }^{14}-S T 230$, Inglaterra ${ }^{14}-S T 9$ y España ${ }^{9 v}$ ST156, entre otros (28).

El Tn3872 se determinó en 9,8 \% de los aislamientos estudiados; este es un elemento genético compuesto que consiste en Tn917, el cual transporta el gen erm(B) insertado en el Tn916 (29). Este transposón fue el más frecuente en aislamientos con diferentes serotipos (6B, 14, $23 \mathrm{~F}$ ) recuperados de muestras nasofaríngeas en Venezuela, y relacionados con varios clones internacionales (30). El mismo estudio identificó otros transposones en portadores sanos, lo cual subraya la importancia del estado del portador nasofaríngeo como reservorio de marcadores de resistencia y fuente de diseminación de clones y transposones de $S$. pneumoniae que pueden causar infecciones neumocócicas invasivas.

El Tn2010 se identificó principalmente en aislamientos con tipo capsular 19A relacionados con el ST320 del complejo 'clonal' CC271. Este transposón se caracteriza por transportar los genes erm(B) y $\operatorname{mef}(\mathrm{E})$ debido a la adquisición del gen erm(B) en aislamientos del clon Taiwán ${ }^{19 F}$-ST236. Se ha planteado la hipótesis de que el CC271 ha emergido como un grupo 'clonal' exitoso a partir un linaje del clon Taiwán ${ }^{19 F}$-ST236 que contiene el Tn2010 y presentó reemplazo del tipo capsular como respuesta a la presión selectiva de la vacuna conjugada heptavalente (31-33). El Tn2010 ha sido reportado en Estados Unidos (33), China $(32,34)$ y Venezuela $(30)$. El CC271 ha emergido como un clon predominante entre los aislamientos con serotipo 19A y su asociación con Tn2010 destaca su importancia en la diseminación de la multirresistencia en muchas regiones del mundo, incluido Colombia.

Nuestros resultados concuerdan con el hecho de que el elemento mega se puede encontrar insertado en diferentes sitios del cromosoma $y$ en aislamientos que pertenecen a diferentes serotipos y grupos 'clonales' $(23,35)$. El elemento mega carece de las enzimas requeridas para la transposición (36) y se disemina por transferencia horizontal entre los diferentes aislamientos de $S$. pneumoniae (23), lo que explica la diversidad de serotipos que transportan dicho elemento genético en los aislamientos estudiados.

Nuestros resultados demuestran que los transposones tienen gran impacto en la difusión de resistencia a macrólidos por medio de la transferencia del determinante de resistencia codificado en el gen erm(B), y contribuyen a la diseminación de marcadores de resistencia a otros antibióticos. Además, la selección y la dispersión de clones que transportan estos transposones pueden cambiar la epidemiología de $S$. pneumoniae y afectar la eficacia de medidas de control tales como la prevención con vacunas conjugadas antineumocócicas.

\section{Agradecimientos}

Se agradece la participación de los integrantes de los laboratorios de salud pública por el envío de los aislamientos de $S$. pneumoniae en el contexto 
de la vigilancia nacional por el laboratorio del proyecto Sistema de Redes de Vigilancia de los Agentes Bacterianos Causantes de Neumonías y Meningitis (SIREVAII) de la Organización Panamericana de la Salud.

\section{Conflicto de intereses}

Los autores declaran no tener ningún conflicto de intereses.

\section{Financiación}

Este trabajo fue financiado por el Instituto Nacional de Salud de Colombia.

\section{Referencias}

1. World Health Organization. Pneumococcal vaccines WHO position paper-2012. Wkly Epidemiol Rec. 2012;87:129-44 http://dx.doi.org/10.1016/j.vaccine.2012.04.093

2. Cornick JE, Bentley SD. Streptococcus pneumoniae: The evolution of antimicrobial resistance to beta-lactams, fluoroquinolones and macrolides. Microbes Infect. 2012;14: 573-83. http://dx.doi.org/10.1016/j.micinf.2012.01.012

3. Leclercq R, Courvalin P. Resistance to macrolides and related antibiotics in Streptococcus pneumoniae. Antimicrob Agents Chemother. 2002;46:2727-34. http://dx.doi.org/10. 1128/AAC.46.9.2727-2734.2002

4. Varaldo PE, Montanari MP, Giovanetti E. Genetic elements responsible for erythromycin resistance in streptococci. Antimicrob Agents Chemother. 2009;53:343-53. http://dx. doi.org/10.1128/AAC.00781-08

5. Instituto Nacional de Salud. Serotipos y patrones de susceptibilidad antimicrobiana de patógenos de importancia en salud pública. Fecha de consulta: 28 de mayo de 2013. Disponible en http://www.ins.gov.co/tramites-y-servicios/ examenes-de-inter\%C3\%A9s-en-salud-publica/Microbiologa/ microbiologia\%20spn\%202012.pdf.

6. Reyes J, Hidalgo M, Díaz L, Rincón S, Moreno J, Vanegas $\mathbf{N}$, et al. Characterization of macrolide resistance in Grampositive cocci from Colombian hospitals: A countrywide surveillance. Int J Infect Dis. 2007;11:329-36. http://dx.doi. org/10.1016/j.ijid.2006.09.005

7. Hidalgo M, Santos C, Duarte C, Castañeda E, Agudelo Cl. Incremento de la resistencia a eritromicina de Streptococcus pneumoniae, Colombia, 1994-2008. Biomédica. 2011;31: 124-31. http://dx.doi.org/10.7705/biomedica.v31i1.343

8. Organización Panamericana de la Salud e Instituto Nacional de Salud de Colombia. Programa de vigilancia de los serotipos y resistencia antimicrobiana de Streptococcus pneumoniae y Haemophilus influenzae. Manual de procedimientos del proyecto. SIREVA II, 2004, p. 41-99. Fecha de consulta: 9 de diciembre de 2011. Disponible en: http:// new.paho.org/hq/index.php?option=com_content\&view=cate gory\&layout=blog\&id=3609\&ltemid=3953\&lang=fr.

9. Clinical Laboratory Standards Institute. MIC testing. Supplemental tables, (M2-A9). Wayne, PA: Clinical Laboratory Standards Institute. 2012.

10. Montanari MP, Mingoia M, Giovanetti. Varaldo PE. Differentiation of resistance phenotypes among erythromycin- resistant pneumococci. J Clin Microbiol. 2001;39:1311-5. http://dx.doi.org/10.1128/JCM.39.4.1311-1315.2001

11. Fasola EL, Bajaksouzian S, Appelbaum PC, Jacobs MR. Variation in erythromycin and clindamycin susceptibilities of Streptococcus pneumoniae by four test methods. Antimicrob Agents Chemother. 1997;41:129-34.

12. Sutcliffe J, Grebe T, Tait-Kamradt A, Wondrack L. Detection of erythromycin-resistant determinants by PCR. Antimicrob Agents Chemother. 1996;40:2562-6.

13. Shiojima T, Fujiki Y, Sagai H, lyobe S, Morikawa A. Prevalence of Streptococcus pneumoniae isolates bearing macrolide resistance genes in association with integrase genes of conjugative transposons in Japan. Clin Microbiol Infect. 2005;11:808-13. http://dx.doi.org/10. 1111/j.1469-0691. 2005.01232.x

14. Brenciani A, Bacciaglia A, Vecchi M, Vitali LA, Varaldo PE, Giovanetti E. Genetic elements carrying erm(B) in Streptococcus pyogenes and association with tet(M) tetracycline resistance gene. Antimicrob Agents Chemother. 2007;51:1209-16. http://dx.doi.org/10.1128/AAC. 01484-06

15. Izdebski R, Sadowy E, Fiett J, Grzesiowski P, Gniadkowski M, Hryniewicz W. Clonal diversity and resistance mechanisms in tetracycline-nonsusceptible Streptococcus pneumoniae isolates in Poland. Antimicrob Agents Chemother. 2007 51:1155-63. http://dx.doi.org/10. 1128/AAC.01384-06

16. Marchese A, Ramírez M, Schito GC, Tomasz A. Molecular epidemiology of penicillin-resistant Streptococcus pneumoniae isolates recovered in Italy from 1993 to 1996. J Clin Microbiol. 1998;36:2944-9.

17. Ayoubi P, Kilic AO, Vijayakumar MN. Tn5253, the pneumococcal omega (cat/tet) BM6001 element, is a composite structure of two conjugative transposons, Tn5251 and Tn5252. J Bacteriol. 1991;173:1617-22.

18. Vela MC, Fonseca N, Di Fabio JL, Castañeda E. Presence of international multiresistant clones of Streptococcus pneumoniae in Colombia. Microb Drug Resist 2001;7:15364. http://dx.doi.org/10.1089/10766290152045020

19. Gentile A, Bardach A, Ciapponi A, García-Martí S, Aruj P, Glujovsky D, et al. Epidemiology of community-acquired pneumonia in children of Latin America and the Caribbean: A systematic review and meta-analysis. Int $\mathrm{J}$ Infect Dis. 2012;16:e5-15. http://dx.doi.org/10.1016/j.jij.2011.09.013

20. Benavides JA, Ovalle OO, Salvador GR, Gray S, Isaacman D, Rodgers GL. Population-based surveillance for invasive pneumococcal disease and pneumonia in infants and young children in Bogotá, Colombia. Vaccine. 2012;30:5886-92. http://dx.doi.org/10.1016/j.vaccine.2012.03.054

21. Montúfar EF, Varón FA, Giraldo LF, Sáenz OA, Rodríguez A, Alarcón AM, et al. Recomendaciones para el diagnóstico, tratamiento y prevención de la neumonía adquirida en la comunidad en adultos inmunocompetentes. Infectio. 2013;17(Supl.1):1-38.

22. Chancey ST, Zhou X, Zähner D, Stephens DS. Induction of efflux-mediated macrolide resistance in Streptococcus pneumoniae. Antimicrob Agents Chemother. 2011;55:341322. http://dx.doi.org/10.1128/AAC.00060-11

23. Del Grosso $\mathbf{M}$, lannelli $\mathbf{F}$, Messina $\mathbf{C}$, Santagati $\mathbf{M}$, Petrosillo N, Stefani S, et al. Macrolide efflux genes mef(A) and $m e f(\mathrm{E})$ are carried by different genetic elements in 
Streptococcus pneumoniae. J Clin Microbiol. 2002;40:774-8. http://dx.doi.org/10.1128/JCM.40.3.774-778.2002

24. Henderson-Begg SK, Roberts AP, Hall LM. Diversity of putative Tn5253-like elements in Streptococcus pneumoniae. Int J Antimicrob Agents. 2009;33:364-7. http:// dx.doi.org/10.1016/j.ijantimicag.2008.10.002

25. Courvalin P, Carlier C. Tn1545: A conjugative shuttle transposon. Mol Gen Genet. 1987;206:259-64.

26. Montanari MP, Cochetti I, Mingoia M, Varaldo PE. Phenotypic and molecular characterization of tetracyclineand erythromycin-resistant strains of Streptococcus pneumoniae. Antimicrob Agents Chemother. 2003;47:223641. http://dx.doi.org/10.1128/AAC.47.7.2236-2241.2003

27. Marimón JM, Pérez-Trallero E, Ercibengoa M, González A, Fenoll A, Spanish Pneumococcal Infection Study Network (G03/103). Molecular epidemiology and variants of the multidrug-resistant Streptococcus pneumoniae Spain ${ }^{14}-5$ international clone among Spanish clinical isolates. J Antimicrob Chemother. 2006;57:654-60. http://dx.doi.org/10. 1093/jac/dkl028

28. Calatayud L, Ardanuy C, Tubau F, Rolo D, Grau I, Pallarés $\mathbf{R}$, et al. Serotype and genotype replacement among macrolide-resistant invasive Pneumococci in adults: Mechanisms of resistance and association with different transposons. J Clin Microbiol. 2010;48:1310-6. http://dx.doi. org/10.1128/JCM.01868-09

29. McDougal LK, Tenover FC, Lee LN, Rasheed JK, Patterson JE, Jorgensen $\mathrm{JH}$, et al. Detection of Tn917-like sequences within a Tn916-like conjugative transposon (Tn3872) in erythromycin-resistant isolates of Streptococcus pneumoniae. Antimicrob Agents Chemother. 1998;42:2312-8

30. Quintero B, Araque M, van der Gaast-de Jongh C, Hermans PW. Genetic diversity of Tn916-related transposons among drug-resistant Streptococcus pneumoniae isolates colonizing healthy children in Venezuela. Antimicrob Agents Chemother. 2011;55:4930-2. http://dx.doi.org/10.1128/AAC. 00242-11

31. Del Grosso M, Northwood JG, Farrell DJ, Pantosti A. The macrolide resistance genes $\operatorname{erm}(\mathrm{B})$ and $\operatorname{mef}(\mathrm{E})$ are carried by Tn2010 in dual-gene Streptococcus pneumoniae isolates belonging to clonal complex CC271. Antimicrob Agents Chemother. 2007;51:4184-6. http://dx.doi.org/10.1128/AAC. 00598-07

32. Li Y, Tomita H, Lv Y, Liu J, Xue F, Zheng B, et al. Molecular characterization of erm(B)- and mef(E)-mediated erythromycin-resistant Streptococcus pneumoniae in China and complete DNA sequence of Tn2010. J Appl Microbiol. 2011;110:254-65. http://dx.doi.org/10.1111/j.1365-2672.2010. 04875.x

33. Bowers JR, Driebe EM, Nibecker JL, Wojack BR, Sarovich DS, Wong AH, et al. Dominance of multidrug resistant CC271 clones in macrolide-resistant Streptococcus pneumoniae in Arizona. BMC Microbiol. 2012;18:12. http:// dx.doi.org/10.1186/1471-2180-12-12

34. Zhou L, Ma X, Gao W, Yao KH, Shen AD, Yu SJ, et al. Molecular characteristics of erythromycin-resistant Streptococcus pneumoniae from pediatric patients younger than five years in Beijing, 2010. BMC Microbiol. 2012;9:12:228. http://dx.doi.org/10.1186/1471-2180-12-228

35. Wierzbowski, AK, Swedlo D, Boyd D, Mulvey M, Nichol KA, Hoban DJ, et al. Molecular epidemiology and prevalence of macrolide efflux genes $\operatorname{mef}(\mathrm{A})$ and $\operatorname{mef}(\mathrm{E})$ in Streptococcus pneumoniae obtained in Canada from 1997 to 2002. Antimicrob. Agents Chemother. 2005;49:1257-61. http://dx.doi.org/10.1128/AAC.49.3.1257-1261.2005

36. Gay K, Stephens DS. Structure and dissemination of a chromosomal insertion element encoding macrolide efflux in Streptococcus pneumoniae. J Infect Dis. 2001;184:56-65. http://dx.doi.org/10.1086/321001 
Cuadro suplementario 1. Genes empleados para la identificación presuntiva de elementos genéticos móviles en aislamientos colombianos de Streptococcus pneumoniae, 1994 - 2011. Disponible en versión en línea.

\begin{tabular}{|c|c|c|c|c|c|c|c|c|c|c|c|}
\hline \multicolumn{11}{|c|}{ Genes } & \multirow[t]{2}{*}{ Presunto transposón } \\
\hline erm(B) & mef(E) & $\operatorname{mef}(\mathrm{A})$ & $\operatorname{tet}(\mathrm{M})$ & cat & aph3'-III & $\begin{array}{c}\text { int } \\
\text { Tn916 }\end{array}$ & $\begin{array}{c}\text { xis } \\
\text { Tn916 }\end{array}$ & $\begin{array}{c}\text { tnpA } \\
\text { Tn917 }\end{array}$ & $\begin{array}{c}\text { tnpR } \\
\text { Tn917 }\end{array}$ & $\begin{array}{c}\text { Int } \\
\text { Tn5252 }\end{array}$ & \\
\hline+ & & & + & + & & + & + & & & + & Tn5253 \\
\hline+ & & & + & + & & + & + & + & + & + & Tn5253 + Tn917 \\
\hline+ & & & + & & & + & + & + & + & & Tn3872 \\
\hline+ & & & + & & + & + & + & & & & Tn1545 \\
\hline+ & & & + & & & + & + & & & & Tn6002 \\
\hline+ & & & + & & & & & & & & Tn1116 \\
\hline \multirow[t]{2}{*}{+} & & & & & + & + & + & + & + & & SpnAp3ermB \\
\hline & & & + & & & + & + & & & & Tn916 \\
\hline \multirow[t]{4}{*}{+} & + & & + & & & + & + & & & & Tn2010 \\
\hline & + & & & & & & & & & & Elemento Mega \\
\hline & + & & + & & & + & + & & & & Tn2009 \\
\hline & & + & & & & & & & & & Tn1207.1 \\
\hline
\end{tabular}

+ : presencia del gen 


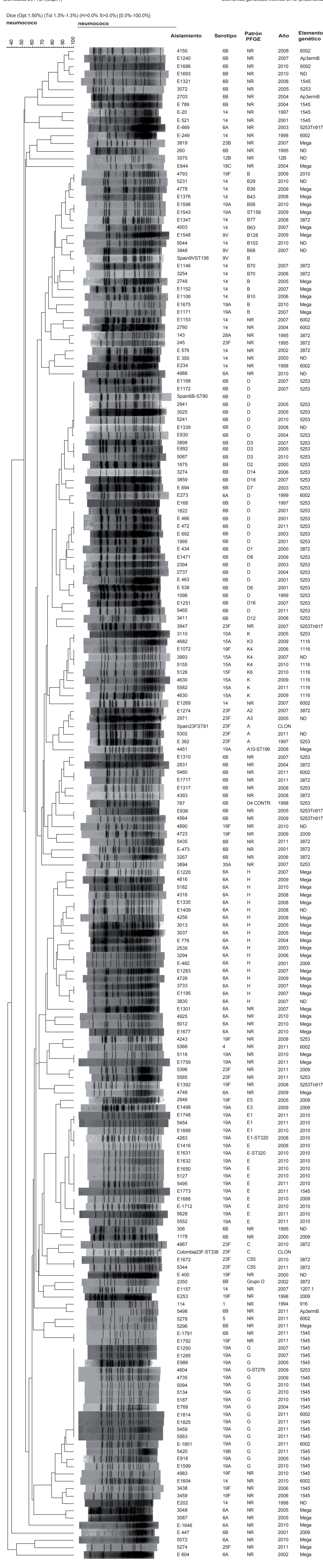

